\title{
WEAK SEQUENTIAL CONVERGENCE \\ IN THE DUAL OF A BANACH SPACE DOES NOT IMPLY NORM CONVERGENCE
}

\author{
BY BENGT JOSEFSON
}

Communicated by Shlomo Sternberg, June 4, 1974

In this note we shall outline a proof of the following result (details will appear elsewhere).

For every infinite-dimensional Banach space $E$ there is a sequence in $E^{\prime}$, the dual space, which tends to 0 in the weak topology $\sigma\left(E^{\prime}, E\right)$ but not in the norm topology. This is well known for separable or reflexive Banach spaces. See also [3] for other examples. The theorem has one of its main applications in the theory of holomorphic functions on infinite-dimensional topological vector spaces [6].

Let $L(E, F)$ denote the set of all bounded linear mappings from $E$ into a Banach space $F$. Let $l^{\infty}$ be the Banach space of all bounded sequences $z=\left(z_{j}\right)_{j=1}^{\infty}$ and $C_{0}$ the Banach space $C_{0}=\left\{z \in l^{\infty}: z_{j} \rightarrow 0\right.$ as $\left.j \rightarrow \infty\right\}$.

TheOREM. There are $\varphi_{j} \in E^{\prime}, j \in \mathbf{N}$, such that $\left\|\varphi_{j}\right\|=1$ and $\lim _{j \rightarrow \infty} \varphi_{j}(z)=0$ for all $z \in E$.

Proof. Let $F \subset E$ be a separable, infinite-dimensional subspace. From [1] and [2] it follows that there are $z^{(j)} \in F$ and $\psi_{j} \in E^{\prime}$ such that $\left\|\psi_{j}\right\|=1,\left\|z^{(j)}\right\|=1, \psi_{j}\left(z^{(j)}\right)=1$ and $\lim _{j \rightarrow \infty} \psi_{j}(z)=0$ for every $z \in F$. Let $\psi \in L\left(E, l^{\infty}\right)$ be the mapping $\psi(z)=\left(\psi_{1}(z), \psi_{2}(z), \cdots, \psi_{j}(z), \cdots\right)$. Put $D=\psi\left(\bar{B}_{E}\right)$, where $\bar{B}_{E}$ is the closed unit ball in $E$. Proj $]_{[U]} D \cap \complement B_{0}$ is not compact for any infinite set $U \subset \mathbf{N}$, where $B_{0}$ is the open unit ball in $C_{0}, \complement$ denotes the complement and $\operatorname{Proj}_{[U]}\left(z_{j}\right)_{j=1}^{\infty}=\left(z_{j}^{\prime}\right)_{j=1}^{\infty}$, where $z_{j}=z_{j}^{\prime}$ if $j \in U$ and $z_{j}^{\prime}=0$ if $j \notin U$. Put $N_{U}(z)=\overline{\lim }_{j, k \rightarrow \infty ; j, k \in U}\left|z_{j}-z_{k}\right|$ for $z \in l^{\infty}$

We shall say that $E$ has property $\mathrm{A}$ if there are linear functionals as in the theorem.

AMS (MOS) subject classifications (1970). Primary 46B99. 
LEMMA 1. If $E$ does not have property $\mathrm{A}$ there exist an infinite set $V \subset \mathbf{N}$ and a number $\epsilon>0$ such that $\sup _{z \in D} N_{U}(z)>\epsilon$ for every infinite $U \subset V$.

The lemma follows easily from the fact that $\operatorname{Proj}_{[U]} D$ is not compact, hence, if $E$ does not have property $\mathrm{A}, \operatorname{Proj}_{[U]} D$ is not separable for any infinite $U \subset \mathbf{N}$.

Lemma 2. If there exist $\varphi_{n} \in L\left(l^{\infty}, \mathbf{C}^{n}\right)$ and $C_{k}>0$ such that $\sup _{z \in D}\left|\operatorname{Proj}_{[t]} \varphi_{n}(z)\right| \geqslant 1$ for every $n \in \mathbf{N}$ and $t \in\{1,2, \cdots, n\}$, and such that for every $z \in D, n \in \mathbf{N}$ and $k \in \mathbf{N},\left|\operatorname{Proj}_{[s]} \varphi_{n}(z)\right| \geqslant 2^{-k}$ for at most $C_{k}$ different $s \in\{1,2, \cdots, n\}$, then $E$ has property $\mathrm{A}$.

The lemma follows essentially from the fact that there are uncountably many $g_{\alpha} \in U_{1} \times U_{2} \times \cdots \times U_{n} \times \cdots$, where $U_{n}=\{1,2, \cdots, n\}$, such that $\operatorname{Proj}_{[n]} g_{\alpha_{1}}=\operatorname{Proj}_{[n]} g_{\alpha_{2}}$ for at most finitely many $n \in \mathrm{N}$ if $\alpha_{1} \neq \alpha_{2}$.

We shall recall the following

THEOREM (Rosenthal [7]). There exists a surjection $\varphi \in L\left(l^{\infty}, l^{2}(B)\right)$, where card $B=2^{\text {card N }}$ and $l^{2}(B)$ is the Hilbert space on $B$.

We shall also recall the fact that if $\varphi^{\prime} \in L\left(F, l^{\infty}\right)$, where $F \subset G$ is a subspace of a Banach space $G, \varphi^{\prime}$ can be extended to $\varphi^{\prime \prime} \in L\left(G, l^{\infty}\right)$ such that $\left\|\varphi^{\prime \prime}\right\|=\left\|\varphi^{\prime}\right\|$, by the Hahn-Banach theorem.

From this fact, the theorem of Rosenthal, and Lemmas 1 and 2, it is possible to prove

Lemma 3. Assume $E$ does not have property A. Then there exist $\varphi_{n} \in L\left(l^{\infty}, l^{2}(B)\right), H_{n} \subset B, z^{(n)} \in D$ and $X_{n}>0$ such that $B \backslash H_{n}$ is finite, $H_{n} \subset H_{n-1} \subset \cdots \subset H_{0}=B$, $\sup _{z \in D}\left\|\operatorname{Proj}_{\left[H_{k-1}\right]} \varphi_{n}(z)\right\| \leqslant X_{k}$ if $k \leqslant n$, $\left\|\operatorname{Proj}_{\left[H_{k-1} \backslash H_{k}\right]} \varphi_{n}\left(z^{(k)}\right)\right\|>X_{k} / 100$ if $k \leqslant n$ and $\left(X_{k}\right)_{k=1}^{\infty}$ is not dominated by a convergent geometric series.

To prove the theorem we suppose that $E$ does not have property $\mathrm{A}$. Then the sequence $\left(X_{n}\right)$ in Lemma 3 may be taken to be decreasing; hence there exists, to every $k \in \mathbf{N}$, a number $n_{k} \in \mathbf{N}$ such that $X_{n_{k}} / X_{n_{k}+k}<1$ $+1 / k$. But then

$$
\left(\frac{100}{X_{n_{k}+k}} \operatorname{Proj}_{\left[H_{n_{k}-1} H_{n_{k}+k-1}\right]} \varphi_{n_{k}+k}\right)_{k=1}^{\infty}
$$

and $C_{n}=2^{2 n} \cdot 200$ have the same properties as $\left(\varphi_{k}\right)_{k=1}^{\infty}$ and $C_{n}$ in 
Lemma 2, which is a contradiction. Q.E.D.

Let $H(F)$ be the set of holomorphic functions, that is to say locally bounded and analytic in the sense of Gâteaux, on a complex locally convex space $F$. A set $B \subset F$ is said to be bounding if $\sup _{z \in B}|f(z)|<\infty$ for every $f \in H(F)$.

COROllaRY 1. If $F$ is an infinite-dimensional, Hausdorff complex locally convex space, then every bounding set has an empty interior.

Proof. The Corollary follows from the theorem if we argue as in [2].

The results in this paper were announced in May 1973 at an international conference on infinite-dimensional holomorphy in Lexington, Kentucky.

\section{REFERENCES}

1. G. Coeuré, Fonctions plurisousharmoniques sur les espaces vectoriels topologiques et applications à l'étude des fonctions analytiques, Ann. Inst. Fourier (Grenoble) 20 (1970), fasc. 1, 361-432. MR 43 \#564.

2. S. Dineen, Unbounded holomorphic functions on a Banach space, J. London Math. Soc. (2) 4 (1971/72), $461-465$. MR 45 \#5753.

3. E. Lacey, Separable quotients of Banach spaces, An. Acad. Brasil. Ci. 44 (1972).

4. J. Lindenstrauss, Some aspects of the theory of Banach spaces, Advances in Math. 5 (1970), 159-180. MR 43 \#5288.

5. L. Nachbin, Topology on spaces of holomorphic mappings, Ergebnisse der Mathematik und ihrer Grenzgebiete, Band 47, Springer-Verlag, New York, 1969. MR 40 \#7787.

6. - Recent developments in infinite dimensional holomorphy, Bull. Amer. Math. Soc. 79 (1973), 625-640.

7. H. Rosenthal, On quasi-complemented subspaces of Banach spaces, with an appendix on compactness of operators from $L^{p}(\mu)$ to $L^{r}(\nu)$, J. Functional Analysis 4 (1969), 176-214. MR 40 \#3277.

8. E. Thorp and R. Whitley, Operator representation theorems, Illinois J. Math. 9 (1965), 595-601. MR 31 \#6126.

DEPARTMENT OF MATHEMATICS, UPPSALA UNIVERSITY, SYSSLOMANSGATAN 8, UPPSALA, SWEDEN 